\title{
Fluorescent Ligands of Kv1 Channels on the Basis of Hongotoxin: Atto488- Hongotoxin
}

\author{
Nikita Orlov ${ }^{1,3^{*}}$, Oksana Nekrasova ${ }^{2}$ and Alexey Feofanov ${ }^{2,3}$ \\ 1. Biological Faculty, Shenzhen MSU-BIT University, Shenzhen, China. \\ 2. Shemyakin-Ovchinnikov Institute of Bioorganic Chemistry, Russian Academy of Sciences, Moscow, \\ Russia. \\ 3. Biological Faculty, Lomonosov Moscow State University, Moscow, Russia. \\ * Corresponding author: n.orlov858@yandex.ru
}

Development of fluorescently labeled peptide toxins, highly efficient and selective ligands of potassium $(\mathrm{K}+)$ channels, is an actual demand for different applications ranging from drug-screening techniques to in vitro and in vivo fluorescent imaging of $\mathrm{K}+$ channels. Hongotoxin 1 ( $\mathrm{HgTx} 1)$, the 39-aa peptide toxin from the venom of scorpion Centruroides limbatus, is a potent blocker of potassium voltage-gated Kv1 - Kv3 channels. Its mutant forms, either radiolabeled ( $\left.{ }^{125} \mathrm{I}-\mathrm{HgTX} 1-\mathrm{A} 19 \mathrm{Y} / \mathrm{Y} 37 \mathrm{~F}\right)$ or fluorescently labeled (HgTX1-A19C) were used to probe localization in tissues [1], pharmacology [2] and subunit organization of $\mathrm{Kv}$-channels [3].

Here we report on the properties of a new fluorescent $\operatorname{HgTx} 1$ derivative labelled with Atto488 fluorophore at the N-terminus (A-HgTx1), which was produced by a chemical synthesis (Smartox Biotechnology, France). To study Kv1-channel binding properties of A-HgTx1, we used a bioengineered analytical system based on the KcsA-Kv1.x $(x=1,3,6)$ chimeric proteins, which carry ligand-binding sites of the corresponding eukaryotic channels Kv1.1, Kv1.3, and Kv1.6 [4-6]. These KcsA-Kv1.x $(x=1,3,6)$ expressed in E.coli cells embed into cell membrane, expose ligand-binding sites into periplasmic space and bind ligands when cells are transformed to spheroplasts. Measurement and quantitative treatment of fluorescence signals associated with spheroplasts in the presence of A-HgTx1 was performed using confocal laser scanning microscopy as described elsewhere [4-6].

We found that A-HgTx1 binds to KcsA-Kv1.1 and KcsA-Kv1.3 and can be displaced from the complexes by an excess of non-labelled recombinant HgTx1 (Fig.1, A). Levels of saturated binding of A-HgTx1 to spheroplasts bearing KcsA-Kv1.1 and KcsA-Kv1.3 are different in accordance with different levels of membrane presentation of these chimeric proteins [7]. A-HgTx1 binding to KcsAbearing spheroplasts or to spheroplasts without channels is low. These results suggest that interactions of A-HgTx1 with KcsA-Kv1.1 and KcsA-Kv1.3 are reversible and specific. Staining of KcsA-Kv1.6bearing spheroplasts by A-HgTx1 is very weak, comparable to the staining of spheroplasts without channels and is not sensitive to the presence of HgTx1. It indicates an absence of interaction between A-HgTx1 and KcsA-Kv1.6 in the tested range of peptide concentrations.

To characterize affinity of A-HgTx1 to Kv1.1- and Kv1.3-binding sites, dissociation constants $\left(\mathrm{K}_{\mathrm{d}}\right)$ were determined. Binding of A-HgTx1 to KcsA-Kv1.1 and KcsA-Kv1.3 is concentration-dependent and saturable. In the case of KcsA-Kv1.1 the A-HgTx1 binding curve has to be corrected for the level of non-specific binding, while in the case of KcsA-Kv1.3 the contribution of non-specific binding is negligible. The Kd values of A-HgTx 1 complexes with Kv1.1- and Kv1.3-binding sites were measured to be $44 \pm 6 \mathrm{nM}$ and $8.4 \pm 0.7 \mathrm{nM}$, respectively. It should be noted that $\mathrm{K}_{\mathrm{d}}$ values for A-HgTx1 are $c a$. 
tenfold lower than those of ${ }^{125} \mathrm{I}-\mathrm{HgTx} 1-\mathrm{A} 19 \mathrm{Y} / \mathrm{Y} 37 \mathrm{~F}$ determined with a radioligand assay [8]. This can be related to a bulk effect of an organic dye used for fluorescent labeling.

It can be concluded that A-HgTx1 is suitable for the search for and study of Kv1.1- and Kv1.3- channel blockers using analytical systems based on the KcsA-Kv1.x $(\mathrm{x}=1,3)$ chimeric proteins as well as for in vitro and in vivo fluorescent imaging of the corresponding Kv1-channels.

\section{References:}

[1] B Pragl et al., Bioconjug. Chem. 13 (2002), p. 416.

[2] G Freudenthaler et al., Histochem. Cell Biol. 117 (2002), p. 97.

[3] A Koschak et al., J. Biol. Chem. 273 (1998), p. 2639.

[4] OV Nekrasova et al., J Neuroimmune Pharmacol. 12 (2017), p. 260.

[5] AI Kuzmenkov et al., J Biol. Chem. 290 (2015), p. 12195.

[6] KS Kudryashova et al., Anal. Bioanal. Chem. 405 (2013), p. 2379.

[7] OV Nekrasova et al., J. Neuroimmune Pharmacol. 4 (2009), p. 83.

[8] C Legros et al., Biochemistry 41 (2002), p.15369.
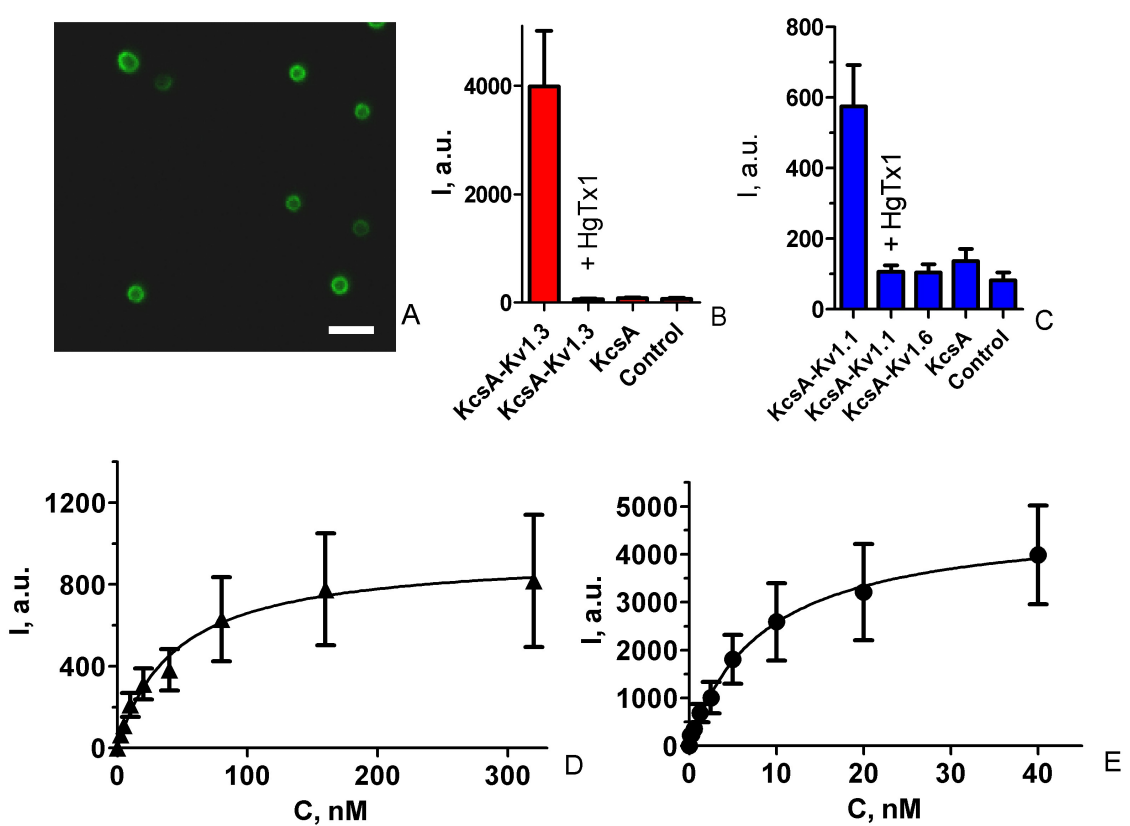

Figure 1. Interactions of A-HgTx1 with spheroplasts bearing KcsA-Kv1.x (x=1, 3) hybrid channels. (A) An example of the confocal fluorescence image showing staining of KcsA-Kv1.3-bearing spheroplasts with A-HgTx1. Bar is $4 \mu \mathrm{m}$. (B) Average fluorescence intensity of A-HgTx1 (40 nM) bound to spheroplasts bearing either KcsA-Kv1.3 or KcsA and to spheroplasts without channels (Control). HgTx1 (500 nM) competes with A-HgTx1 for the binding to KcsA-Kv1.3. (C) Average fluorescence intensity of A-HgTx1 (150 nM) bound to spheroplasts bearing KcsA-Kv1.1, KcsA-Kv1.6 or KcsA and spheroplasts without channels (Control). HgTx1 (1000 nM) competes with A-HgTx1 for the binding to KcsA-Kv1.1. (D, E) Concentration dependent binding of A-HgTx1 to spheroplasts bearing KcsA-Kv1.1 (D) or KcsA-Kv1.3 (E). 